\title{
Conjugated Polymers Combined with a Molecular Beacon for Label-Free and Self-Signal-Amplifying DNA Microarrays
}

\author{
By Kangwon Lee, Jean-Marie Rouillard, Bong-Gi Kim, Erdogan Gulari, and \\ Jinsang Kim*
}

A conjugated polymer (CP) and molecular-beacon-based solid-state DNA sensing system is developed to achieve sensitive, label-free detection. A novel conjugated poly(oxadiazole) derivative exhibiting amine and thiol functional groups (POX-SH) is developed for unique chemical and photochemical stability and convenient solid-state on-chip DNA synthesis. POX-SH is soluble in most nonpolar organic solvents and exhibits intense blue fluorescence. POX-SH is covalently immobilized onto a maleimidofunctionalized glass slide by means of its thiol group. Molecular beacons having a fluorescent dye or quencher molecule as the fluorescence resonance energy transfer (FRET) acceptor are synthesized on the immobilized POX-SH layer through direct on-chip oligonucleotide synthesis using the amine side chain of POX-SH. Selective hybridization of the molecular beacon probes with the target DNA sequence opens up the molecular beacon probes and affects the FRET between POX-SH and the dye or quencher, producing a sensitive and label-free fluorescence sensory signal. Various molecular design parameters, such as the size of the stem and loop of the molecular beacon, the choice of dye, and the number of quencher molecules are systematically controlled, and their effects on the sensitivity and selectivity are investigated.

\section{Introduction}

DNA microarrays have become a powerful tool in gene sequencing and gene expression studies. ${ }^{[1-3]}$ Principally, DNA microarrays are collections of microscopic spots of oligonucleotides, commonly representing many different kinds of single genes, arrayed on a microscope slide or a chip by covalent attachment for high-throughput screening. Thousands of individual genes can be spotted or directly synthesized on a single square inch slide at a time. Analyte DNA or RNA molecules are then labeled with a fluorescent dye and spread out onto the DNA microarray. Complementary sequences will be hybridized with the probing DNA on the DNA microarray. After washing off nonspecifically bound analyte DNA, the microarray is scanned to visualize the bound DNA by means of the labeled fluorescent dye. The fluorescent dye labeling is necessary even though dye tagging the analyte DNA requires time and money because conventional DNA microarrays do not have any capability to generate sensory signal itself. In this regard, a selfsignaling DNA microarray that allows label-free detection is highly desirable. There have been several new generations of responsive probes having optical or electrical signal upon binding with a labelfree target. Among these are surface plasmonic resonance (SPR), ${ }^{[4]}$ quartz crystal microbalance, ${ }^{[7-13]}$ label-free electronic detection of DNA, ${ }^{[14-15]}$ the use of intercalating dye, ${ }^{[16-19]}$ and molecular beacon concept. ${ }^{[20-25]}$

The molecular beacon was devised as a self-signaling probe to eliminate the time consuming fluorescence labeling of analyte DNAs. A traditional molecular beacon is a hairpin-structured single-stranded DNA (ssDNA), in which a fluorescent dye and a quencher molecule located at the two ends of the hairpin are in close contact. Due to the adjacent quencher molecule, the dye cannot emit any fluorescence unless the molecular beacon hybridizes with a complementary ssDNA. Hybridization with a complementary ssDNA opens the hairpin, moving the fluorescent dye away from the quencher, thus allowing the dye to emit its fluorescence signal. 


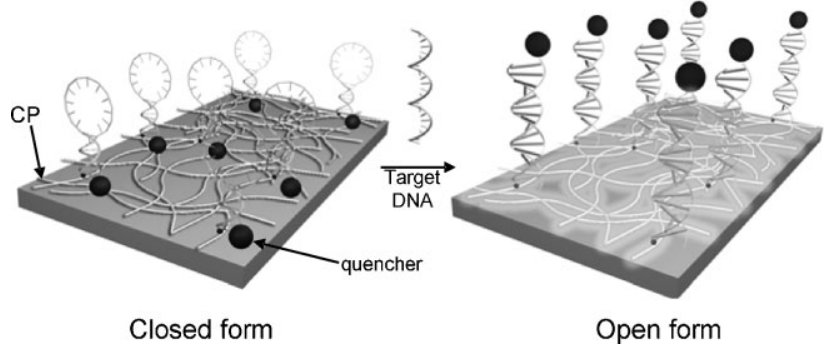

Figure 1. Schematic representation of the label-free and signal-amplifying DNA microarray composed of conjugated polymers and molecular beacons.

Conjugated polymers (CPs) have received much attention as signal-transducing elements for sensory applications due to the signal amplification property and the versatility in molecular design of CPs. Various chemical sensors and biosensors have been developed based on CPs. ${ }^{[26-42]}$ Previously we developed signalamplifying molecular DNA sensors to achieve selective and sensitive detection of DNA in aqueous solution. We also extended the signal-amplifying strategy to the DNA microarrays by uniquely combining rationally designed chemically and photochemically stable CPs and the solid-state DNA synthesis. ${ }^{[19,41,42]}$

In this contribution, we systematically investigated the molecular design principle of DNA molecular beacons to add a self-signaling feature to the signal-amplifying CP-based DNA microarrays to achieve label-free DNA detection (Fig. 1). We covalently tethered a newly developed CP by using its thiol side chain to a maleimide-modified solid substrate and then synthesized molecular beacons from the amine functional group of the polymer through on-chip DNA synthesis. The molecular beacon probes were terminated by a quencher or a fluorescent dye to produce turn-on-type and turn-off-type self-signaling and signal-amplifying DNA microarrays. We systematically studied the effects of the variation in the probe size, stem length, and the number of quenchers per molecular beacon probe on the sensitivity of the microarrays. The devised strategy can provide convenient self-signaling and signal amplifing properties and can be readily applied to the conventional DNA microarray fabrication method.

\section{Results and Discussion}

\subsection{Polymer Design and Synthesis}

The chemical structure of the polymer was carefully designed by considering the required properties for covalent immobilization of the polymer, as well as the harsh reaction condition of the on-chip DNA synthesis. We previously developed an oxadiazole-containing and aminefunctionalized CP. ${ }^{[41,43]}$ The oxadiazole unit had provided chemical and photochemical stability while the amine side chain had immobilized the polymer to a solid substrate and the on-chip DNA synthesis at the same time. We used the same oxadiazole unit for stability, but we added another unit having a thiol, solely for the polymer immobilization, in order to control the amount of the amine-containing unit for the on-chip DNA synthesis. The synthesis of the monomer M1, which is required for the thiol-containing poly(oxadiazole) derivative (POX-SH), is illustrated in Scheme 1 (detailed synthesis and characterization of the compounds are provided in the Experimental Section). 2,5-Dibromohydroquinone was reacted with 1,6dibromohexane by Williamson-ether synthesis. To prevent the formation of crosslinked by-products between the dibromoalkyl compound and hydroquinone, the latter was very slowly added dropwise into an excess of dibromohexane solution. However from a later separate investigation, we found out that if the dibromoalkane molecule has a longer chain than a $\mathrm{C}_{5}$ when it is attached to the phenol group, the reactivity of the other bromine at the other end of the alkyl chain is significantly decreased. Therefore, Williamson-ether synthesis of a dibromohexyl compound with a phenolic group can be done in a one-pot synthesis with a reasonable yield by using excess amounts of the dibromoalkyl compound. The resulting compound 1 was reacted with thiourea to give the dithiol compound 2. This compound turned out to be very unstable in air due to disulfide formation. Therefore, the thiol group was protected using trityl chloride. Initially, the mixture was translucent due to the limited solubility of compound 2 in methylene chloride. However, the solution became completely transparent after the thiol group was protected with the trityl group.

As shown in Scheme 2, the copolymerization was carried out by means of Suzuki crosscoupling polymerization of the aryl halide monomers M1, M2, and M3 and a fluorine monomer M4 having a borolene unit in the presence of a palladium catalyst. ${ }^{[44]}$ Monomer M2 having amine groups and M3 having the oxadiazole unit were prepared according to our previous work. ${ }^{[41,43]}$ The monomers M1, M2, and M3 showed good solubility in organic solvents, such as chloroform and tetrahydrofuran (THF) $\left(>15 \mathrm{mg} \mathrm{mL}^{-1}\right.$ ). We controlled the monomer ratio to obtain CPs having various compositions. As summarized in Table 1, there is a strong inverse correlation between the molecular weight of the resulting polymers and the fraction of M1 in the aryl halide monomers. It is likely that the polymerization rate decreases once $\mathbf{M 1}$, which has a bulky side chain, is incorporated in the growing polymer chain. The number $\left(M_{\mathrm{n}}\right)$ and weight $\left(M_{\mathrm{w}}\right)$ average molecular weight of the polymers were determined by gel permeation chromatography (GPC) using monodisperse polystyrenes as standards. The $M_{\mathrm{n}}$ and the $M_{\mathrm{w}}$ of the polymers before the cleavage of the 

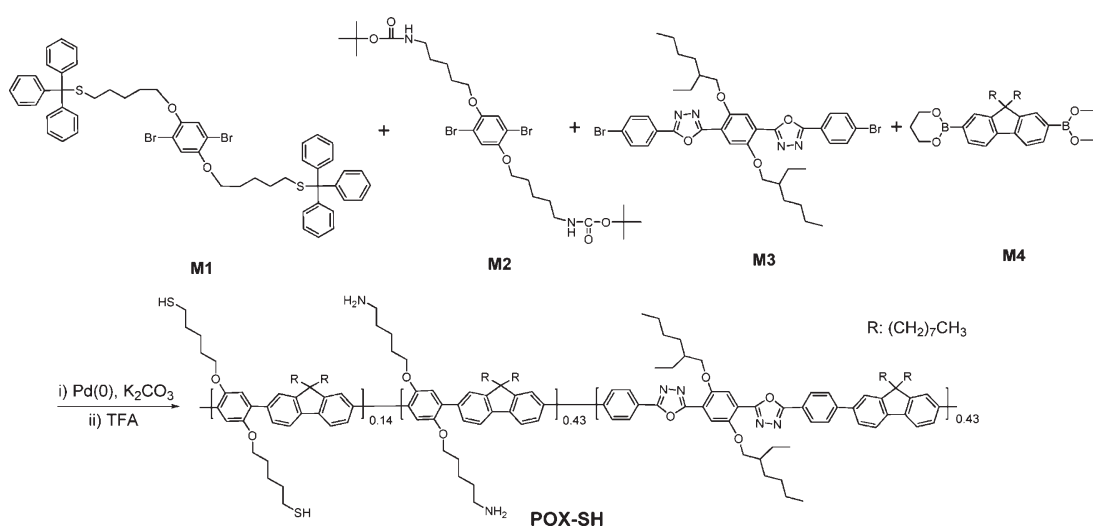

Scheme 2. Polymerization of POX-SH.

Table 1. The monomer ratio and molecular weight of the resulting polymers.

\begin{tabular}{cccccc}
\hline M1 & M2 & M3 & M4 & $M_{\mathrm{n}}[\mathrm{a}]$ & $M_{\mathrm{w}}[\mathrm{a}]$ \\
\hline 1 & 1 & 1 & 3 & 2700 & 15400 \\
1 & 2 & 2 & 5 & 4700 & 23000 \\
1 & 3 & 3 & 7 & 19500 & 76000 \\
\hline
\end{tabular}

[a] Unit: $\mathrm{g} \mathrm{mol}^{-1}$, molecular weight is calculated by polystyrene-based GPC with THF as eluent.

protecting groups are listed in Table 1 . The polymer before the deprotection of the tert-butyl carbamate ( $t$-BOC) and trityl groups was favorably soluble in nonpolar organic solvents, like chloroform and THF, but insoluble in polar solvents and had a pale yellow color.

Deprotection of the trityl and $t$-BOC groups was conducted in $50 \%$ trifluoroacetic acid (TFA) in chloroform to give POX-SH. The cleavage of the trityl and $t$-BOC groups was monitored by ${ }^{1} \mathrm{H}$-NMR analysis. For the $t$-BOC cleavage, two peaks at 4.50 and $1.43 \mathrm{ppm}$ corresponding to the carbamate proton and tertiary butyl proton, respectively, disappeared, and a new single peak at $1.97 \mathrm{ppm}$ corresponding to the primary amine was observed. In addition, a peak corresponding to the alkyl protons next to the $-\mathrm{NH}$-group shifted from 3.13 to $2.67 \mathrm{ppm}$. For the trityl group cleavage, the peak intensities at $7.40-7.20 \mathrm{ppm}$ corresponding to the aromatic protons significantly decreased, and we observed new peaks at 2.55 and $1.35 \mathrm{ppm}$ corresponding to the alkyl and free thiol protons, respectively. After the deprotection, the solubility of POX-SH in chloroform slightly decreased, but significantly decreased in THF.

\subsection{Fabrication of the DNA Microarray}

The procedure of the polymer immobilization on a glass substrate is schematically described in Figure 2. In order to prevent any loss of the polymer during the on-chip DNA synthesis, POX-SH $\left(M_{n}=19500\right)$ was covalently tethered to a glass substrate. Amine groups were first introduced onto the glass by means of a self-assembled monolayer of aminopropyltrimethoxysilane (APTMS). The subsequent treatment of the slide with the heterofunctional crosslinker, succinimidyl 4-[N-maleimidomethyl]cyclohexane-1-caroboxylate (SMCC) gave the maleimido group, which has a high affinity to thiol. POX-SH was then covalently linked onto the glass substrate through the maleimido group. After immobilization of POX-SH, any unbound polymer was removed by cycles of sonication and thorough rinsing.

Surface characterization of the glass substrate was performed by water contact angle measurements and UV-vis spectroscopy (see Fig. S1 and S2 in the Supporting Information for details). The water contact angle gradually increased with the APTMS and SMCC reactions followed by the polymer tethering because of the increased hydrophobicity of the surface, confirming successful polymer tethering. The UV-vis spectrum of the substrate after the SMCC reaction shows a broad tailing band around $300 \mathrm{~nm}$ corresponding to SMCC suggesting successful conjugation of SMCC onto the amine-modified surface. The resulting POX-SH-coated slide showed two new bands at 355 and $385 \mathrm{~nm}$ corresponding to the $0-1,0-0$ band of POX-SH absorption, respectively. The intense blue photoluminescence at $425 \mathrm{~nm}$ as shown in Figure 3 also confirms the successful POX-SH tethering to the glass substrate.

The DNA beacon probes were synthesized on the slide having POX-SH by applying the phosphoramidite chemistry to the amine group of POX-SH. The density of the DNA beacon probes after the on-chip DNA synthesis was estimated by measuring the amount of dimethyltrityl (DMT), which was cleaved from the synthesized oligonucleotides at the final cycle of the on-chip DNA synthesis.
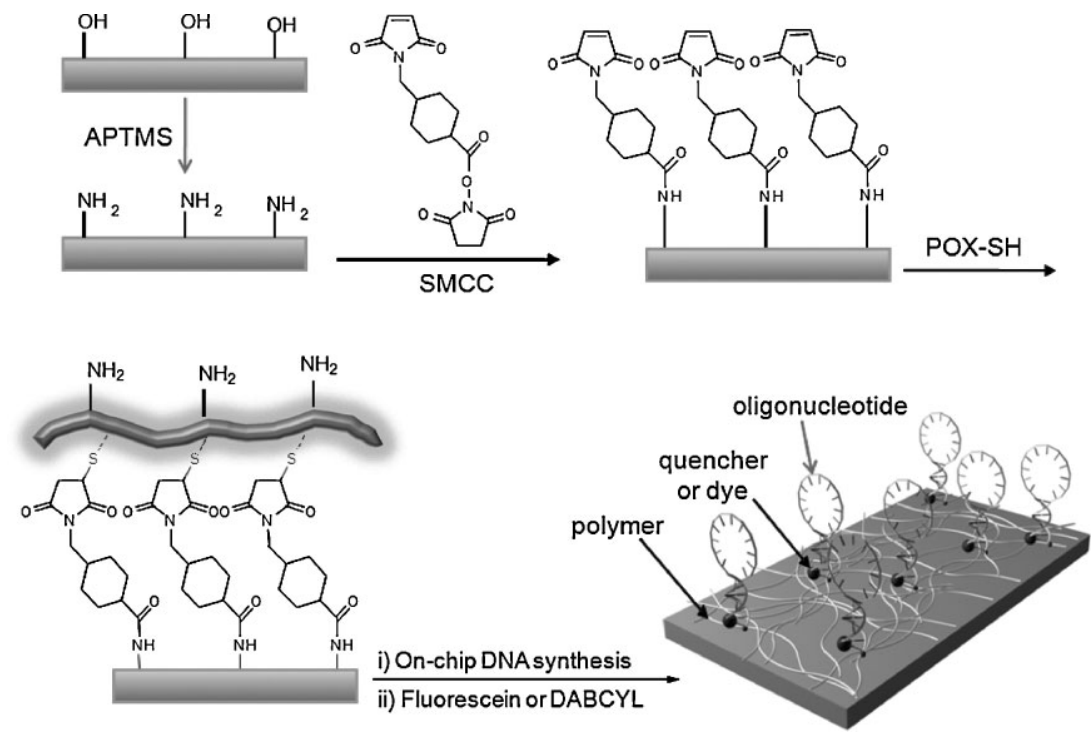

Figure 2. Surface modification and polymer immobilization. 


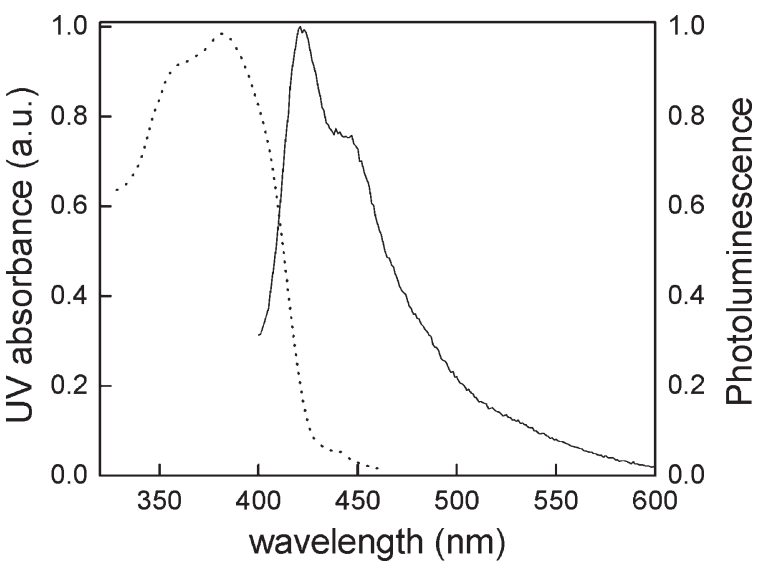

Figure 3. UV absorption (dotted) and fluorescence emission spectra (solid) of the glass slide having covalently linked POX-SH.

The surface concentration of the molecular beacon probes was consistently $2.8 \times 10^{12}$ molecules $\mathrm{cm}^{-2}$. This value is smaller than the surface density $\left(5.0 \times 10^{13}\right.$ molecules $\left.\mathrm{cm}^{-2}\right)$ of oligonucleotides of conventional DNA microarrays presumably because the amine density of the POX-SH layer is smaller than that of the highly packed amine groups of conventional microarrays.

\subsection{Molecular Beacon Design and its Effects on the Sensitivity}

We designed turn-on-type molecular beacon probes, as well as turn-off-type molecular beacon probes. The turn-on-type probes have 4-(4-dimethylaminophenylazo)benzoic acid (DABCYL) as a quencher at the end of the sequence so that the fluorescent emission of POX-SH is quenched when the molecular beacon forms the closed hairpin structure. The quenched emission will be turned-on upon binding with a target DNA sequence. The turn-offtype probes have a fluorescence dye instead of a quencher so that efficient fluorescence resonance energy transfer (FRET) from POX-SH to the dye makes the dye produce amplified emission. The amplified emission from the dye will be turned-off when the probe hybridizes with its target sequence. We systematically varied the lengths of the loop and stem and the amounts of DABCYL or fluorescent dye in the molecular beacon design, and investigated the effects of these parameters on the sensitivity of the DNA microarray.

Table 2 shows the sequence of the molecular beacon probes, P1 and P2, which have different lengths, and their target comple- mentary ( $\mathrm{C} 1$ and $\mathrm{C} 2)$ and noncomplementary sequences ( $\mathrm{N} 1$ and N2). The underlined sequences are for the formation of the closed hairpin stem in the absence of the target DNA sequence. The shaded sections of the molecular probes are the sequences complementary to the target DNA sequences. Even though the two specific DNA sequences were used as a probe sequence in this study, various DNA sequences can be simultaneously synthesized on the POX-SH layer for high-throughput detection. We first investigated the turn-on-type probes. The probe P1 sequence adapted from the Staphylococcus aureus femA gene $\mathrm{e}^{[45]}$ was synthesized on the POX-SH layer and terminated by DABCYL attachment. The successful on-chip synthesis of the molecular beacon having DABCYL was confirmed by the new DABCYL absorption band at $460 \mathrm{~nm}$ in the UV-vis absorption spectrum (Fig. 4). We added two thymine (T) units as a spacer between the P1 sequence and the POX-SH to allow for sufficient mobility of the molecular beacon enabling efficient hybridization. Therefore, there are three T units between the POX-SH and the stem of P1, and the distance is $10.2 \AA$ as depicted in Figure 5 a. In this case the POX-SH emission was quenched $52 \%$ by DABCYL while the emission was fully recovered when the complementary DNA sequence was introduced. The control slide having the same P1 sequence, but no quencher did not show any fluorescence quenching in the closed form, confirming that the $52 \%$ quenching was induced by DABCYL (Fig. 5b). Because the sensitivity of this type of turn-on sensors is determined by the level of the emission intensity recovery relative to the initial intensity, the initial emission intensity of POX-SH should be quenched as much as possible. Then DABCYL should be located close to the POX-SH surface to sufficiently quench the POX-SH emission because the FRET efficiency is highly dependent on the inverse sixth power of the intermolecular distance between the donor and accepter. To achieve the closest distance between a DABCYL and a quencher, we removed the TT spacer even though this may reduce the mobility of the molecular beacon. As we can see in Figure 5c, in this case, the initial emission intensity of POX-SH was quenched $48 \%$ more than that of POX-SH having the TTspacer (Fig. 5c) due to the smaller distance between DABCYL and POX-SH. The emission was fully recovered when the complementary DNA sequence was introduced.

We applied super-quenchers (SQs), an array of multiple quenchers, to our DNA microarrays to further suppress the initial fluorescence emission. Tan et al. investigated multiple quenchers in molecular beacon design. The researchers synthetically assembled two or three DABCYLs at the end of a molecular beacon and achieved better quenching efficiency in its closed form and relatively large enhancement of fluorescent turn-on signal in solution due to the super-quenching effect. ${ }^{[4]}$ We synthesized two

Table 2. The sequence of the DNA hairpin probes, $\mathrm{P} 1$ and $\mathrm{P} 2$, and their target complementary (C1 and $\mathrm{C} 2$ ) and noncomplementary DNAs (N1 and $\mathrm{N} 2)$ used in this study. The shaded sequences are the complementary sections of the probes toward the targets.

\begin{tabular}{|c|c|}
\hline Name & Sequence \\
\hline P1 (probe) & $5^{\prime}$-DABCYL or dye-CCA CGC TCA TCA TAA CCT TCA GCA AGC TTT AAC TCA TAG TGA GCG TCG T-3'-glass \\
\hline P2 (probe) & $5^{\prime}$-DABCYL or dye-CGC TCC TAA AAC GAC GGC CAC TGG AGC GT-3'-glass \\
\hline $\mathrm{Cl}$ (P1 complement) & $5^{\prime}$-ACG CTC ACT ATG AGT TAA AGC TTG CTG AAG GTT ATC A-3' \\
\hline C2 (P2 complement) & 5'-ACT GGC CGT CGT TTT AGG AGC G-3' \\
\hline N1 (random sequence) & $5^{\prime}$-TGA GAA TCA TAC TCA ATT TCG AAC GAC TTC CAA TAC T-3' \\
\hline N2 (random sequence) & $5^{\prime}$-TCA CAA ACA GCA AAA TCC TAA C-3' \\
\hline
\end{tabular}




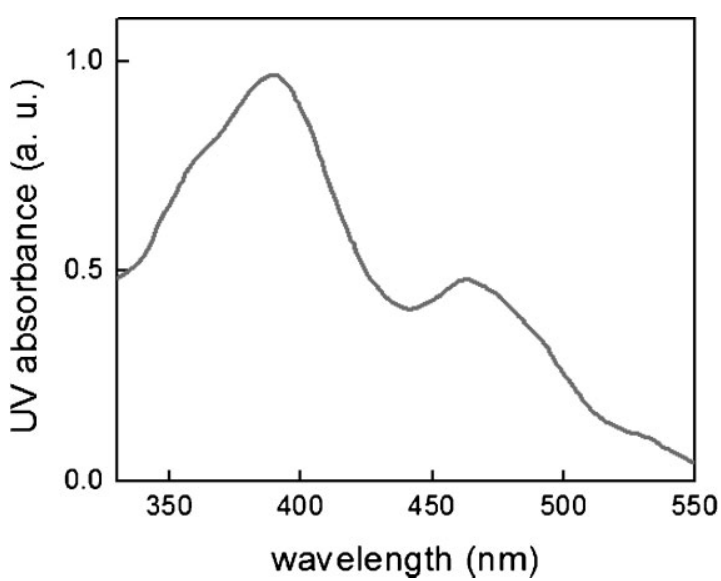

Figure 4. UV-vis absorption spectrum of the DNA microarray after the onchip DNA synthesis of the molecular beacon having DABCYL.

types of super-quenchers (three DABCYLS, 3Q, and nine DABCYLs, 9Q) directly on the POX-SH layer as illustrated in Scheme 3. However, unlike the results of the solution study in the literature, the quenching efficiency of these super-quenchers was even worse than that of the monoquencher. The $3 \mathrm{Q}$ and $9 \mathrm{Q}$ showed only 47 and $57 \%$ quenching efficiency, respectively; this is much less than that of the monoquencher (75\%). This is likely because the molecular beacon containing the super-quenchers does not form a stable closed stem in the solid state due to the steric hindrance of the bulky super-quencher at the solid surface.

We also investigated the effect of the loop size of the molecular beacon on the initial quenching of the closed form. We tested the longer P1 of $50 \mathrm{bp}$ and the shorter P2 of $30 \mathrm{bp}$ listed in Table 1 . As we can see from Figure 6, while the emission of both DNA microarrays having P1 and P2, respectively, was completely recovered upon hybridization with the target DNA sequences, C1 and $\mathrm{C} 2$, respectively, the initial emission intensity of POX-SH was better quenched in the case of the shorter $\mathrm{P} 2$ probe. The yield of the on-chip DNA synthesis to add each base unit for the molecular beacon synthesis is over $99 \%$. Therefore, the overall synthetic yield of the longer P1 of $50 \mathrm{bp}$ and the shorter P2 of $30 \mathrm{bp}$ is approximately 60.5 and $74.0 \%$, respectively. This calculation suggests that the density of DABCYL of P2 is higher than that of P1. This is presumably the reason for the better quenching efficiency of the shorter probe $\mathrm{P} 2$.

In addition to the turn-on strategy discussed above, we extended the molecular-beacon-based label-free and signal-amplifying DNA-microarray concept to a turn-off sensor scheme by incorporating a fluorescent dye instead of the DABCYL quencher (Fig. 7). A fluorescein (green dye) or a HEX (yellow dye), which act as FRET acceptors, was placed at the end of the molecular beacon. In the closed form, the fluorescent dye is located close to the POXSH layer and efficiently takes the fluorescence resonance energy from the FRET donor, POX-SH. The emission signal from the dye is largely amplified (turned-on) through the FRET process because macromolecular POX-SH absorbs a much larger number of photons than a small molecular dye and the absorbed energy can be effectively transferred to the dye. When the molecular beacon probe recognizes its target sequence, it forms a stable probe-target double helix by DNA hybridization. This recognition event moves the dye away from the POX-SH layer preventing FRET from the donor POX-SH to the dye, the FRET acceptor. Therefore, the dye emission should be quenched as a result (turned-off).

Figure 8 shows the fluorescence spectra of POX-SH having the molecular probe $\mathrm{P} 1$ or $\mathrm{P} 2$ with a fluorescein dye (5'-fluorescein-CCA CGC TCA TCA TAA CCT TCA GCA AGC TTT AAC TCA TAG TGA GCG TGG T-3') before and after hybridization with the complementary DNA sequence. Figure 8a shows the signal amplification of the fluorescein dye of the closed form of P1. While the direct excitation of the fluorescein at $490 \mathrm{~nm}$ produced a weak emission, the selective excitation of POX-SH at $380 \mathrm{~nm}$ generated much stronger emission from the fluorescein. Upon binding with the complementary DNA (C1), the emission of the fluorescein decreased (solid triangles) due to the prevention of FRET between POX-SH and the fluorescein while the emission from fluorescein decreased only slightly (solid circles) in the presence of noncomplementary DNA (N1) (Fig. 8b). As shown in Figure $8 \mathrm{c}$ and d, P2 having the shorter sequence $\left(5^{\prime}\right.$-fluorescein-CGC TCC TAA AAC GAC GGC CAG TGG AGC GT$3^{\prime}$ ) showed a slightly better amplification and emission quenching upon hybridization with the target sequence (C2), such as in the turn-on cases.

We also used HEX instead of fluorescein. The absorption and emission of HEX are at longer wavelengths compared to those of fluorescein,
Figure 5. Emission spectra of POX-SH with various molecular beacons before (squares) and after hybridization (triangles) with the target sequence. a) P1 having the TT spacer and a DABCYL, b) P1 having the TT spacer but without a DABCYL, c) P1 having a DABCYL but without the TT spacer, and d) P1 having three DABCYLs and without the TT spacer. 

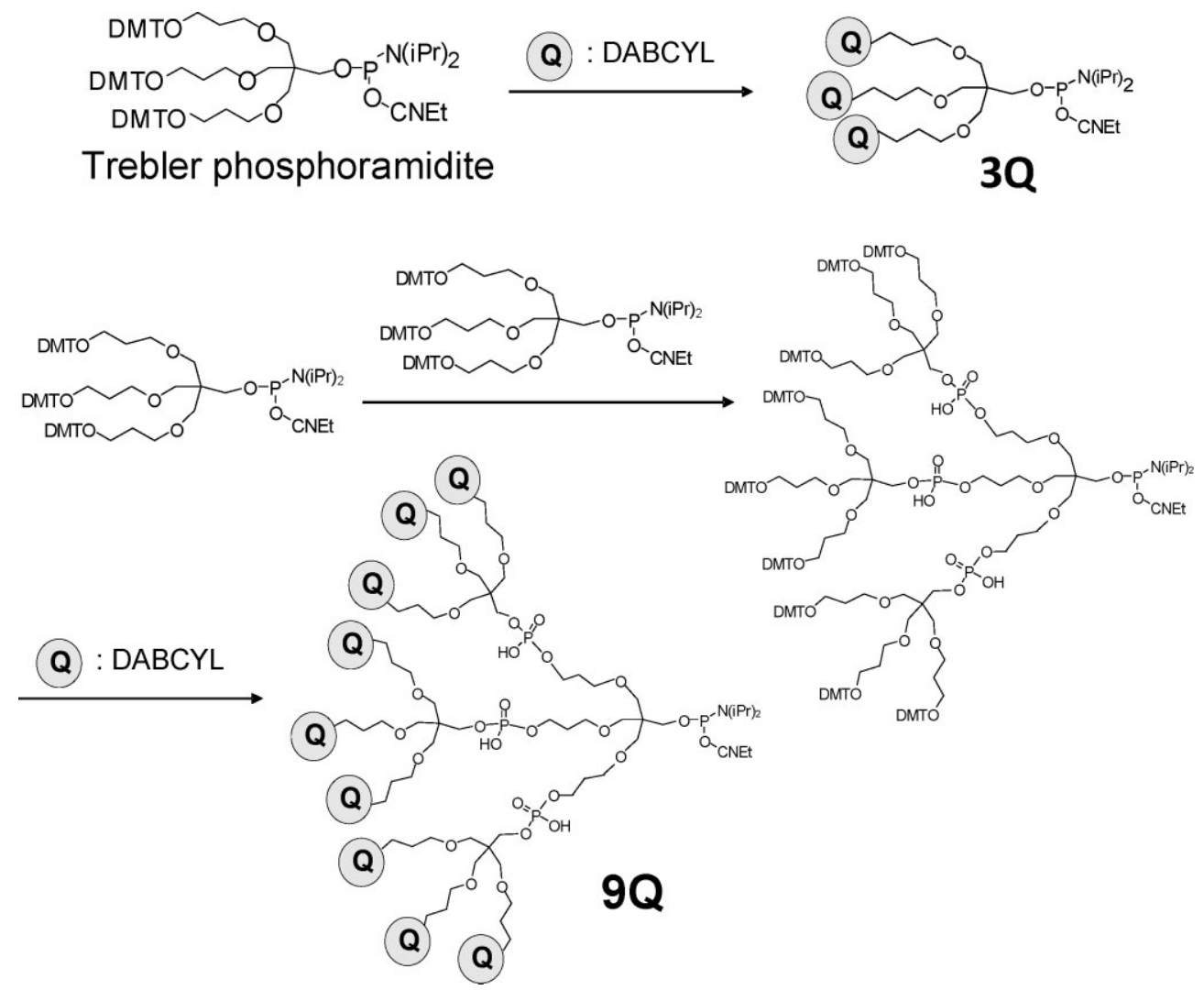

Scheme 3. Super-quencher synthesis using trebler phosphoramidite (DMT=4,4'-diemethoxytrityl, Et=ethyl).

making the dye emission more discrete from the POX-SH emission. Even though the emission intensity of HEX is stronger, the overall degree of amplification and the signal quenching by hybridization in the HEX system are similar to those of the fluorescein system (Fig. 9).

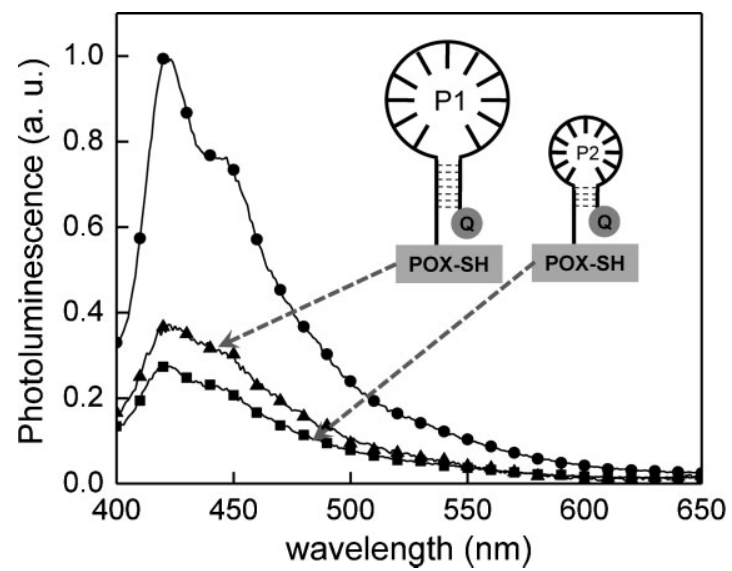

Figure 6. Photoluminescence spectra of the DNA microarray having two different probes, P1 (triangles) and P2 (squares), upon hybridization with the target complementary sequences, $\mathrm{C} 1$ and $\mathrm{C} 2$, respectively.

\section{Conclusion}

We designed and synthesized multifunctional CP POX-SH having the oxadiazole unit for stability, a thiol group for covalent tethering to a solid substrate, and an amine unit for the solid-state on-chip DNA synthesis. We developed self-signaling DNA microarray by synthesizing molecular beacons directly on the POX-SH layer by applying the on-chip DNA synthesis. A turn-on system with molecular beacon probes having a nonfluorescent quencher (DABCYL) and a turn-off system with a fluorescent dye (fluorescein or HEX) were developed. The turn-on sensor case, the fluorescent signal of the POX-SH layer originally quenched by DABCYL was fully recovered upon binding with the complementary DNA while a nonspecific DNA sequence resulted in a minimal change in the fluorescence emission. We controlled the lengths of the loop and stem and the number of DABCYL in the molecular beacon design and investigated the effects of these design parameters on the sensitivity of the DNA microarray. A smaller distance between the beacon and the POX-SH layer, a smaller size loop, and a monoquencher provided the best sensitivity. In the turn-off system, a fluorescein (green dye) or a HEX (yellow dye) acting as FRET acceptors was placed at the end of the molecular beacon. In the closed form, the fluorescent dye located close to the POX-SH layer, allowed efficient fluorescence resonance energy from the FRETdonor, POX-SH, and produced amplified emission. When the molecular probe recognized its target sequence, it formed a stable probe-target double helix by DNA hybridization. 


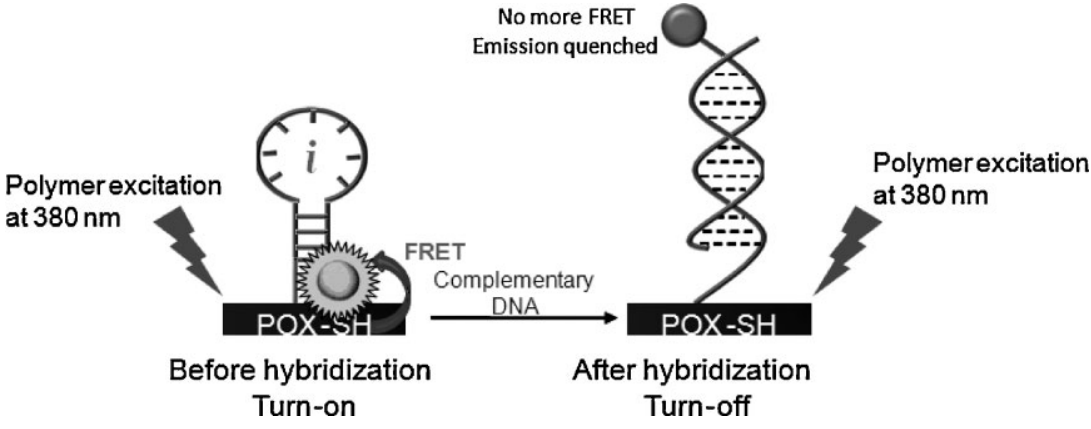

Figure 7. Schematic representation of the turn-off sensor by combining a fluorescence dye with a molecular beacon.
This recognition event moved the dye away from the POX-SH layer preventing FRET from the donor POX-SH to the dye and quenched the dye emission. We demonstrated the selfsignaling and signal-amplifying DNA microarray by combining rational molecular design of an emissive $\mathrm{CP}, \mathrm{POX}$-SH, and solid-state DNA synthesis of molecular beacons. The obtained results provide a molecular design principle to build a self-signaling DNA microarray and control its sensitivity.


Figure 8. Photoluminescence spectra of POX-SH having $\mathrm{P} 1$ or $\mathrm{P} 2$ probe with a fluorescein dye. Prehybridization state emission of a) P1 and c) P2 when excited at $380 \mathrm{~nm}$ (squares) and $490 \mathrm{~nm}$ (triangles). The emission intensity changes of the fluorescein emission of b) P1 and d) P2 after hybridization with the target complement ( $\mathrm{C} 1$ and $\mathrm{C} 2$, triangles) and random DNA (N1 and N2, circle), respectively (excitation wavelength: $380 \mathrm{~nm})$.
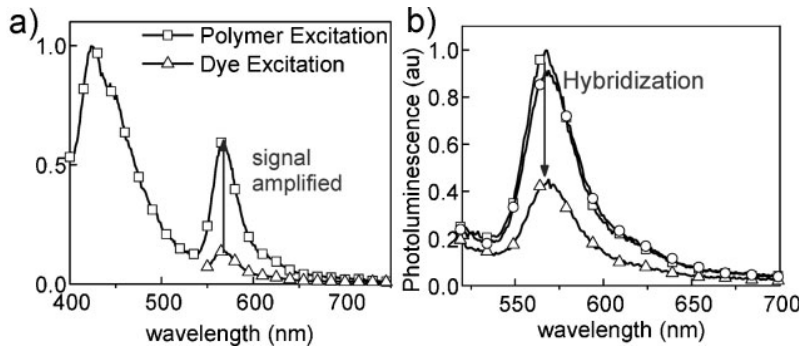

Figure 9. Photoluminescence spectra when HEX dye was used. a) Emission of the microarray before hybridization when excited at $380 \mathrm{~nm}$ (squares) and $535 \mathrm{~nm}$ (triangles). (b) The emission intensity change of HEX after hybridization with the target DNA (triangles) and noncomplementary DNA (circles).

\section{Experimental}

Materials and Methods: All solvents and reagents for monomer and polymer syntheses were used as received from Sigma-Aldrich Chemical Co. or Fischer Scientific Co. All the chemicals for the on-chip oligonucleotide synthesis such as phosphoramidites, 5'-DABCYL phosporamidite, $5^{\prime}$-hexachlorofluorescein phosphoramidite, and trebler phosphoramidite were purchased from Glen Research Co. A crosslinker, SMCC, was purchased from Pierce Inc. (Fisher Scientific Co.).

${ }^{7} \mathrm{H}$ NMR spectra ( 400 or $500 \mathrm{MHz}$ ) were obtained with Varian Inova 400 or 500 NMR instrumentation. UV-vis absorption spectra were obtained using a Varian Cary50 UV-vis spectrophotometer. Photoluminescence spectra and quantum yield in the solid state were recorded using PTI QuantaMaster spectrofluorometer equipped with an integrating sphere. The $M_{n}, M_{w}$, and the molecular weight distributions were determined by GPC with respect to polystyrene standards (Waters Corp.) using THF as an eluent.

Synthesis of 1,4-Dibromo-2,5-bis(6-bromohexyloxy)benzene (1): 2,5Dibromohydroquinone $(13.8 \mathrm{~g}, 51.5 \mathrm{mmol}), 1,6$-dibromohexane $(151 \mathrm{~g}$, $618 \mathrm{mmol}$ ), potassium carbonate $(56.9 \mathrm{~g}, 412 \mathrm{mmol}$ ), and $600 \mathrm{~mL}$ of acetone were added to a $1000 \mathrm{~mL}$ two-neck round bottom flask. The mixture was stirred at $50^{\circ} \mathrm{C}$ for $72 \mathrm{~h}$. The crude mixture was filtered to remove any insoluble salt and impurities. The solution was concentrated at reduced pressure and precipitated in $800 \mathrm{~mL}$ of methanol. The product isolated by filtration and dried in vacuo. Additional purification was done by recrystallization in a chloroform and hexane mixture. Yield: (16.8 g, 55\%), ${ }^{1} \mathrm{H}-\mathrm{NMR}\left(500 \mathrm{MHz}, \mathrm{CDCl}_{3}\right): \delta / \mathrm{ppm} 7.00(\mathrm{~s}, 2 \mathrm{H}$, aromatic), $3.96(\mathrm{t}, 4 \mathrm{H}$, $\left.-\mathrm{OCH}_{2}\right), 3.43\left(\mathrm{t}, 4 \mathrm{H},-\mathrm{CH}_{2} \mathrm{Br}\right), 1.97\left(\mathrm{~m}, 4 \mathrm{H},-\mathrm{CH}_{2}-\right), 1.92(\mathrm{~m}, 4 \mathrm{H}$, $\left.-\mathrm{CH}_{2}-\right), 1.52\left(\mathrm{~m} \mathrm{4H},-\mathrm{CH}_{2}-\right) \cdot{ }^{13} \mathrm{C}-\mathrm{NMR}\left(125 \mathrm{MHz}, \mathrm{CDCl}_{3}\right): \delta / \mathrm{ppm}$ $150.1,118.7,111.3,69.8,33.9,32.5,28.9,27.7,27.3$.

Synthesis of 6,6'-(2,6-Dibromo-1,4-phenylene)bis(oxy)dihexane-7-thiol (2): Compound $1(8.22 \mathrm{~g}, 13.8 \mathrm{mmol})$ was dissolved in $70 \mathrm{~mL}$ of ethanol and stirred for $30 \mathrm{~min}$ under purging argon. Thiourea $(2.11 \mathrm{~g}, 27.6 \mathrm{mmol})$ was slowly added to the solution, and the solution was heated under reflux overnight. The crude mixture was poured into $10 \%$ sodium hydroxide solution $(50 \mathrm{~mL})$ and stirred for $4 \mathrm{~h}$ at room temperature. The solution was neutralized with $10 \% \mathrm{HCl}(32 \mathrm{~mL})$. The mixture was filtered at reduced pressure. The solid was extracted with methylene chloride/water three times. The combined organic phase was again washed with deionized (DI) water two times. The methylene chloride solution was dried with sodium sulfate, filtered, and evaporated at reduced pressure at room temperature. The compound was additionally dried in vacuo. The product obtained was unstable in air due to disulfide formation and showed decreased solubility in organic solvents. The product was used in the following step without further purification. Yield $(6.5 \mathrm{~g}, 94 \%),{ }^{7} \mathrm{H}-\mathrm{NMR}\left(500 \mathrm{MHz}, \mathrm{CDCl}_{3}\right): \delta / \mathrm{ppm}$ 7.09 (s, $2 \mathrm{H}$, aromatic), $3.98\left(\mathrm{t}, 4 \mathrm{H},-\mathrm{OCH}_{2}\right), 2.56\left(\mathrm{t}, 4 \mathrm{H},-\mathrm{CH}_{2} \mathrm{Br}\right), 1.83$ $\left(\mathrm{m}, 4 \mathrm{H},-\mathrm{CH}_{2}-\right), 1.67\left(\mathrm{~m}, 4 \mathrm{H},-\mathrm{CH}_{2}-\right), 1.53\left(\mathrm{~m} \mathrm{4H},-\mathrm{CH}_{2}-\right), 1.46(\mathrm{~m}$ $\left.4 \mathrm{H},-\mathrm{CH}_{2}-\right), 1.36(\mathrm{t}, 2 \mathrm{H},-\mathrm{SH})$.

Synthesis of (6'6'-(2,5-Dibromo-1,4-phenylene)bis(oxy)bis(hexane-6, 1diyl))bis(tritylsulfane) (M1): Compound $2(5.00 \mathrm{~g}, 10 \mathrm{mmol}$ ) and $40 \mathrm{~mL}$ 
of anhydrous methylene chloride were added to a $250 \mathrm{~mL}$ two-neck round bottom flask. After stirring the mixture for $10 \mathrm{~min}$ under purging argon, trityl chloride $(11.14 \mathrm{~g}, 40.0 \mathrm{mmol})$ in methylene chloride $(10 \mathrm{~mL})$ was slowly added, and the mixture was stirred under purging argon at room temperature for $24 \mathrm{~h}$. The solution was extracted with $10 \% \mathrm{NaOH}$ and washed again with DI water. The solution was dried over magnesium sulfate, filtered, and concentrated by rotary evaporation at reduced pressure. Additional purification was done by silica gel column chromatography (ethyl acetate:hexane $=1: 9$ ), and the product was dried in vacuo to give a white powder (yield: $7.4 \mathrm{~g}, 75 \%)$. ${ }^{1} \mathrm{H}-\mathrm{NMR}(500 \mathrm{MHz}$, $\mathrm{CDCl}_{3}$ ): $\delta / \mathrm{ppm} 7.42$ ( $\mathrm{m}, 12 \mathrm{H}$, aromatic), 7.30 (dd, $12 \mathrm{H}$, aromatic), 7.22 ( $\mathrm{m}, 6 \mathrm{H}$, aromatic), 7.09 (s, $2 \mathrm{H}$, aromatic), $3.91\left(\mathrm{t}, 4 \mathrm{H},-\mathrm{OCH}_{2}\right), 2.17$ $\left(\mathrm{t}, 4 \mathrm{H},-\mathrm{CH}_{2} \mathrm{Br}\right), 1-50-1.20\left(\mathrm{~m}, 16 \mathrm{H},-\mathrm{CH}_{2}-\right) .{ }^{13} \mathrm{C}-\mathrm{NMR}(125 \mathrm{MHz}$, $\left.\mathrm{CDCl}_{3}\right): \delta / \mathrm{ppm} 150.05,145.06,130.05,127.84,126.55,118.49,111.16$, 70.11, 66.44, 31.91, 28.88, 28.65, 28.52, 25.54.

Polymer Synthesis (POX-SH): M1 (39.3 mg, $39.9 \mu \mathrm{mol})$, M2 (76.4 mg, $119.7 \mu \mathrm{mol})$, M3 $(93.4 \mathrm{mg}, 119.7 \mu \mathrm{mol})$, M4 (140.3 mg, $279.3 \mu \mathrm{mol})$, THF $(7 \mathrm{~mL})$, and $1 \mathrm{M} \mathrm{K}_{2} \mathrm{CO}_{3}(7 \mathrm{~mL})$ were added to a $50 \mathrm{~mL}$ Schlenk flask. Degassed tetrakis(triphenylphosphine) palladium(0) $(6.46 \mathrm{mg}, 5.6 \mu \mathrm{mol})$ in THF $(1 \mathrm{~mL})$, prepared in a separate Schlenk, was transferred to the monomer mixture by cannula, and the monomer solution was degassed by several cycles of vacuum and argon purging. Suzuki-type polymerization was carried out at $75^{\circ} \mathrm{C}$ for $48 \mathrm{~h}$. Polymer solution was precipitated in $100 \mathrm{~mL}$ of methanol and filtered by gravity force. The solid product was washed with water $(2 \times 20 \mathrm{~mL})$ and acetone $(2 \times 20 \mathrm{~mL})$. The polymer pellet was redissolved in chloroform and washed twice with DI water. The solution was dried over $\mathrm{MgSO}_{4}$, filtered, and evaporated using a rotary evaporator at reduced pressure to give the precursor polymers. TFA $(10 \mathrm{~mL})$ was carefully added to polymer in chloroform $(10 \mathrm{~mL})$, and the polymer solution was stirred at room temperature for $8 \mathrm{~h}$ in order to cleave the $t$-BOC and trityl blocking groups. After evaporation of the solvent and TFA, the polymer was redissolved in chloroform and washed with $1 \mathrm{M} \mathrm{KOH}$ solution, followed by $\mathrm{NaCl}$ solution, and $\mathrm{DI}$ water to give a yellow polymer P1 (yield: $180 \mathrm{mg}, 63 \%$ ). The $M_{\mathrm{n}}$ and $M_{\mathrm{w}}$ were calculated for the polymer before the cleavage of $t-\mathrm{BOC}$ due to the limited solubility of $\mathrm{P} 1$ in THF (the GPC eluent): $M_{\mathrm{n}}=19500, M_{\mathrm{w}}=76000$. ' $\mathrm{H}-\mathrm{NMR}\left(500 \mathrm{MHz}, \mathrm{CDCl}_{3}\right)$ :

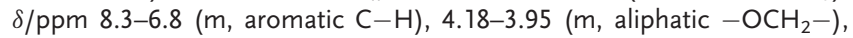
2.75-2.65 (two t, aliphatic $-\mathrm{CH}_{2} \mathrm{SH}$ and $-\mathrm{CH}_{2} \mathrm{NH}_{2}$ ), 2.2-0.6 (broad $\mathrm{m}$, aliphatic $\mathrm{CH}_{2}$ and $\mathrm{CH}_{3}$ ).

Glass Substrate Modification and Polymer Immobilization: A slide glass $(2.54 \mathrm{~cm} \times 7.62 \mathrm{~cm})$ was incubated in ammonium hydroxide/water $/ 35 \%$ hydrogen peroxide $(1: 4: 1 \mathrm{v} / \mathrm{v} / \mathrm{v})$ solution at $80{ }^{\circ} \mathrm{C}$ for $1 \mathrm{~h}$. The slide was rinsed with a sufficient amount of DI water (ca. $50 \mathrm{~mL}$ ) and dried with nitrogen gas. It was dipped into Pirahna solution (caution: $\mathrm{H}_{2} \mathrm{SO}_{4}: 35 \%$ $\mathrm{H}_{2} \mathrm{O}_{2}=3: 7 \mathrm{v} / \mathrm{v}$, highly exothermic, $\mathrm{H}_{2} \mathrm{SO}_{4}$ must be poured slowly into $\mathrm{H}_{2} \mathrm{O}_{2}$ ) and incubated overnight. The slide was then rinsed with $100 \mathrm{~mL}$ of DI water, dried with a stream of nitrogen gas, transferred into 97\% APTMS/ methanol/water $(1: 24: 1 \mathrm{v} / \mathrm{v} / \mathrm{v})$, and sonicated for $30 \mathrm{~min}$. The solution temperature was elevated from 20 to $37^{\circ} \mathrm{C}$. The slide was washed with methanol $(30 \mathrm{~mL})$ and $\mathrm{DI}$ water $(30 \mathrm{~mL})$ and dried with nitrogen gas. It was baked in a convection oven at $120^{\circ} \mathrm{C}$ for $30 \mathrm{~min}$. The aminofunctionalized glass slide was incubated with $7.5 \mathrm{mg}$ of SMCC in anhydrous dimethylformamide (DMF) $(200 \mu \mathrm{L})$ at room temperature for $2 \mathrm{~h}$. Excess SMCC was washed out with $50 \mathrm{~mL}$ of anhydrous DMF and anhydrous chloroform and dried with a stream of air. Only one side of the slide was reacted with POX-SH in chloroform $(9 \mathrm{~mL})$ with a variety of incubation times $(0.5,1,3 \mathrm{~h}$, and overnight) and subsequently washed with chloroform, methylene chloride, and DI water. Further cleaning was conducted by the sonication of the glass slide in chloroform for $5 \mathrm{~min}$ to remove any physically bounded polymer chains to substrate. After drying the slide by a stream of nitrogen, it was preserved in a glovebox $\left(-35^{\circ} \mathrm{C}\right)$ for further DNA synthesis. Slide preparation and POX immobilization have been reported previously [46].

On-Chip Oligonucleotide Synthesis and Oligonucleotide Density Measurement: The light-directed, on-chip oligonucleotide reactions were carried out using apparatus described previously [19, 24, 41, 46, 47, 52]. On-chip oligonucleotide synthesis was performed using standard phosphoramidite chemistry except for the deprotection step, where a
$2 \%$ trichloroacetic acid (TCA)/methylene chloride (single sequence) or iodium PGA-P solution (multisequences by arrayal patterns) to deprotect the terminal dimethoxytrityl protecting group at selected reaction sites. $D A B C Y$, dye (fluorescein or HEX), or trebler phosphoramidite was also used at the end of oligo synthesis to introduce single-/multi-quenchers and dye as an energy acceptor. Oligonucleotide synthesis was confirmed by UV-vis spectra and fluorescence images after hybridization with dyelabeled DNA, obtained from a GenePix 4000B microarray scanner (Molecular Devices Corp.) with dual lasers $(532 \mathrm{~nm} / 17 \mathrm{~mW}, 635 \mathrm{~nm} /$ $10 \mathrm{~mW}$ ). To measure the density of DNA on polymer-coated glass, DMT quantification method was used. A glass slide having oligonucleotide was treated with $0.1 \mathrm{M}$ of $p$-toluenesulfonic acid monohydrate (TSA, $4 \mathrm{~mL}$ ) in anhydrous acetonitrile for $1 \mathrm{~min}$. DMT molecules cleaved from the final cycle of oligo synthesis was collected. UV absorption of the DMT solution was measured by UV spectroscopy to quantify DMT concentration. A major peak corresponding to a DMT cation appears at $500 \mathrm{~nm}$. We used a second peak at $410 \mathrm{~nm}$ with an extinction coefficient of 28690 . According to the Beer's law below, the concentration for a given DMT solution is proportional to UV absorbance and the molar extinction coefficient is constant:

$A=\varepsilon b c$

where $A$ is the absorbance (no units, since $A=\log _{10} P_{0} / P$ ), $\varepsilon$ is the molar absorptivity with units of $\mathrm{L} \cdot \mathrm{mol}^{-1} \cdot \mathrm{cm}^{-1}, b$ is the path length of the sample $(\mathrm{cm}, 1 \mathrm{~cm}$ cuvette), and $c$ is the concentration of the DMT in acetonitrile, expressed in $\mathrm{mol} \cdot \mathrm{L}^{-1}$.

Hybridization Test and Fluorescence Detection: Hybridization buffer contained $20 \mathrm{~mm}$ Tris- $\mathrm{HCl}$ (where Tris is tris(hydroxymethyl)aminomethane), $55 \mathrm{~mm} \mathrm{NaCl}, 10.8 \mathrm{~mm} \mathrm{KCl}, 2 \mathrm{~mm}$ EDTA, and $100 \mathrm{~mm}$ $\mathrm{MgCl}_{2}$ at $\mathrm{pH}$ 8.0. All DNA hybridization tests were conducted at $25^{\circ} \mathrm{C}$. Slides are first rinsed with hot water sufficiently and immersed in hybridization buffer. Hybridization DNA solutions $\left(1 \times 10^{-5} \mathrm{M}\right.$, hybridization buffer above: $49.5 \mu \mathrm{L}$, complementary DNA: $0.5 \mu \mathrm{L}$ ) were heated to $90{ }^{\circ} \mathrm{C}$ to prevent nonspecific loop formation of the target oligo and were applied onto the slide. The slide was covered with blank slide and kept in a humid chamber and incubated for $1 \mathrm{~h}$. The slide was rinsed with blank hybridization buffer and cold water $\left(3^{\circ} \mathrm{C}\right)$ by quick dipping. FRET tests were performed by checking UV absorbance and photoluminescence emission changes before and after hybridization upon the excitation of POX-SH at $380 \mathrm{~nm}$ or the dye excitation at $490 \mathrm{~nm}$ (fluorescein) or $535 \mathrm{~nm}$ (HEX). Relative fluorescence increase in the fluorescein test was calculated using $\left(I_{\text {target }}-I_{\text {polymer }}\right) /\left(I_{\text {probe }}-I_{\text {polymer }}\right)$, where $I_{\text {probe }}$ and $I_{\text {target }}$ are the photoluminescence intensity of dye before and after hybridization, respectively, and $I_{\text {polymer }}$ is the photoluminescence intensity of polymer before DNA synthesis.

\section{Acknowledgements}

We gratefully acknowledge the financial support from the National Science Foundation (Career DMR 064486). J. K. and K. L. also acknowledge Ilju Foundation for the Ilju scholarship. Supporting Information is available online from Wiley InterScience or from the authors.

Received: July 1, 2009 Published online: September 8, 2009

[1] R. F. Service, Science 1998, 282, 396.

[2] G. Ramsay, Nat. Biotechnol. 1998, 16, 40.

[3] E. M. Southern, Trends Genet. 1996, 12, 110.

[4] E. A. Smith, M. Kyo, H. Kumasawa, K. Nakatani, I. Saito, R. M. Corn, J. Am. Chem. Soc. 2002, 124, 6810.

[5] J. M. Brockman, A. G. Frutos, R. M. Corn, J. Am. Chem. Soc. 1999, 121, 8044. 
[6] Y. Chen, K. Munechika, D. S. Ginger, Nano Lett. 2007, 7, 690.

[7] J. Wang, Nucleic Acids Res. 2000, 28, 3011.

[8] Y. Okahata, Y. Matsunobo, K. Ijiro, A. Murakami, M. Makino, J. Am. Chem. Soc. 1992, 114, 8299

[9] A. Bardea, A. Dagan, I. Ben-Dov, B. Amit, I. Willner, Chem. Commun. 1998, 839.

[10] F. Patolsky, A. Lichtenstein, I. Willner, J. Am. Chem. Soc. 2000, 122, 418.

[11] A. P. R. Johnston, E. S. Read, F. Caruso, Nano Lett. 2005, 5, 953.

[12] A. P. R. Johnston, H. Mitomo, E. S. Read, F. Caruso, Langmuir 2006, 22, 3251.

[13] T. H. Nguyen, M. Elimelech, Biomacromolecules 2007, 8, 24.

[14] H. Shiigi, S. Tokonami, H. Yakabe, T. Nagaoka, J. Am. Chem. Soc. 2005, $127,3280$.

[15] S.-J. Park, T. A. Taton, C. A. Mirkin, Science 2002, 295, 1503.

[16] F. He, Y. Tang, M. Yu, F. Feng, L. An, H. Sun, S. Wang, Y. Li, D. Zhu, G. C. Bazan, J. Am. Chem. Soc. 2006, 128, 6764.

[17] T. Maruyama, T. Takata, H. Ichinose, N. Kamiya, H. Kuma, N. Hamasaki, H. Morita, M. Goto, Biotechnol. Prog. 2005, 21, 575.

[18] T. Maruyama, L. C. Park, T. Shinohara, M. Goto, Biomacromolecules 2004, 5, 49.

[19] K. Lee, K. Maisel, J.-M. Rouillard, E. Gulari, J. Kim, Chem. Mater. 2008, 20, 2848.

[20] B. Bockisch, T. Grunwald, E. Spillner, R. Bredehorst, Nucleic Acids Res. 2005, 33, 101.

[21] H. Du, C. M. Strohsahl, J. Camera, B. L. Miller, T. D. Krauss, J. Am. Chem. Soc. 2005, 127, 7932

[22] M. Culha, D. L. Stokes, G. D. Griffin, T. Vo-Dinh, J. Biomed. Opt. 2004, 9, 439.

[23] W. Tan, K. Wang, T. J. Drake, Curr. Opin. Chem. Biol. 2004, 8, 547.

[24] N. E. Broude, Trends Biotechnol. 2002, 20, 249.

[25] G. Goel, A. Kumar, A. K. Puniya, W. Chen, K. Singh, J. Appl. Microbiol. 2005, $99,435$.
[26] For a review of chemical and biological sensors based on conjugated polymers, see S. W. Thomas, III, G. D. Joly, T. M. Swager, Chem. Rev. 2007 107, 1339.

[27] Y. Chen, R. Thakar, P. T. Snee, J. Am. Chem. Soc. 2008, 130, 3744.

[28] Z. Chen, C. Xue, W. Shi, F.-T. Luo, S. Green, J. Chen, H. Liu, Anal. Chem. 2004, 6, 6513.

[29] I.-B. Kim, U. H. F. Bunz, J. Am. Chem. Soc. 2006, 128, 2818

[30] H.-A. Ho, M. Leclerc, J. Am. Chem. Soc. 2004, 126, 1384.

[31] F. He, Y. Tang, S. Wang, Y. Li, D. Zhu, J. Am. Chem. Soc. 2005, 127, 12343

[32] P. H. Kwan, M. J. MacLachlan, T. M. Swager, J. Am. Chem. Soc. 2004, 126, 8638.

[33] H. Jiang, X. Zhao, K. S. Schanze, Langmuir 2006, 22, 5541.

[34] S. W Thomas, III, T. M. Swager, Adv. Mater 2006, 18, 1047.

[35] K. P. R. Nilsson, O. Inganäs, Macromolecules 2004, 37, 9109.

[36] F. He, Y. Tang, M. Yu, F. Feng, L. An, H. Sun, S. Wang, Y. Li, D. Zhu, G. C. Bazan, J. Am. Chem. Soc 2006, 128, 6764.

[37] H. Xu, H. Wu, F. Huang, S. Song, W. Li, Y. Cao, C. Fan, Nucleic Acids Res. 2005, 33, 83

[38] H.-J. Kim, J. Lee, T. Kim, T. S. Lee, J. Kim, Adv. Mater. 2008, 20, 1117.

[39] J. Lee, H.-J. Kim, J. Kim, J. Am. Chem. Soc. 2008, 130, 5010.

[40] F. Feng, Y. Tang, F. He, M. Yu, X. Duan, S. Wang, Y. Li, D. Zhu, Adv. Mater 2007, 19, 3490

[41] K. Lee, J.-M. Rouillard, T. Pham, E. Gulari, J. Kim, Angew. Chem. Int. Ed. 2007, 46, 4667.

[42] K. Lee, L. K. Povlich, J. Kim, Adv. Funct. Mater. 2007, 17, 2580.

[43] K. Lee, H.-J. Kim, J. C. Cho, J. Kim, Macromolecules 2007, 40, 6457.

[44] N. Miyaura, A. Suzuki, Chem. Rev. 1995, 95, 2457.

[45] H. Du, M. D. Disney, B. L. Miller, T. D. Krauss, J. Am. Chem. Soc. 2003, 125 4012.

[46] C. J. Yang, H. Lin, W. Tan, J. Am. Chem. Soc. 2005, 127, 12772.

[47] X. Gao, E. LeProust, H. Zhang, O. Srivannavit, E. Gulari, P. Yu, C. Nishiguchi, Q. Xiang, X. Zhou, Nucleic Acids Res. 2001, 29, 4744. 